\title{
A Spectrophotometric Microtiter-Based Assay of the Ability of UHT-Treated Milk to Reduce XTT
}

\author{
Hiroyuki Ukeda, ${ }^{1}$ Yukihiko Goto, ${ }^{1}$ Masayoshi Sawamura, ${ }^{1}$ Hirozo Kusunose, ${ }^{1}$ Hideaki KamiKadO ${ }^{2}$ and \\ Toshiro KAMEI ${ }^{2}$ \\ ${ }^{1}$ Department of Bioresources Science, Faculty of Agriculture, Kochi University, Monobe B-200, Nankoku 783, Japan \\ ${ }^{2}$ Central Research Institute, Meiji Milk Products Co., Ltd., 1-21-3, Sakae-cho, Higashimurayama-shi, Tokyo 189, Japan
}

Received September 20, 1995

\begin{abstract}
A spectrophotometric microtiter-based assay was established for quantification of the ability of UHT-treated

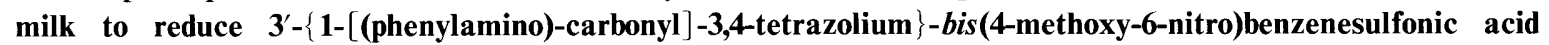
hydrate (XTT). Under the optimized conditions, $40 \mu \mathrm{l}$ of a milk sample was mixed with $60 \mu \mathrm{l}$ of $0.5 \mathrm{mM}$ XTT solution (pH 7.0) saturated with menadione in each well of a 96-well microtiter plate. The formed XTT formazan in the reaction mixture was determined by an increase in the absorbance difference between that at $492 \mathrm{~nm}$ and at 600 $\mathbf{n m}$ during the incubation for $20 \mathrm{~min}$ at ambient temperature. The use of the absorbance at $600 \mathrm{~nm}$ as a reference wavelength permitted the determination of XTT formazan without any interference from the turbidity caused by the addition of the milk sample. The present microtiter plate assay could differentiate two kinds of UHT-treated milk with a different extent of thermal treatment. In addition, the reducibility of the UHT-treated milk gradually decreased during storage dependent on the period or temperature. The method was very convenient and economical; it is promising as a method of estimating the extent of the heat treatment of milk and the storage conditions.
\end{abstract}

Keywords: milk, Maillard reaction, tetrazolium salt, microplate, heat treatment

The Maillard reaction is known to occur in milk during heat treatment (Walstra \& Jenness, 1984; Finot et al., 1981). In the initial stage of this reaction, mainly the $\varepsilon$-amino groups of the essential protein bound-lysine react with lactose to form the so-called "Amadori product." Thus-modified lysine is biologically not available for digestion (Finot et al., 1981). Therefore, it is important from the nutritional standpoint to estimate the extent of the Maillard reaction during the heat treatment.

The change in milk during the Maillard reaction in the heating process can be estimated by a change in hydroxymethylfurfural (HMF) and lactulose concentration (Fink \& Kessler, 1988). Because these concentrations increase with increasing heat treatment, they have been recognized to be the most promising indicators of the treatment (Keeney \& Bassette, 1959; Geier \& Klostermeyer, 1980). However, the determination methods are generally time-consuming and tedious.

The authors have proposed an assay method for determining the ability of UHT-treated milk to reduce XTT as a method of evaluating the extent of the Maillard reaction (Ukeda et al., 1995). Compared with the previously reported methods such as the quantification of lactulose (Geier \& Klostermeyer, 1980), HMF (Keeney \& Bassette, 1959) and furosine (Finot et al., 1981), the XTT reduction assay is a rapid and convenient method and is found to be applicable for estimating not only the extent of thermal treatment but also the storage conditions because the ability decreased dependent on the storage period and temperature (Ukeda et al., 1995). However, a drawback of the method was caused by the fact that the XTT used was a relatively expensive reagent. In order to overcome the drawback, we have established a microtiter plate assay of the ability of UHT-treated milk to reduce XTT in the present investigation.

\section{Materials and Methods}

Reagents Menadione (2-methyl-1,4-naphthoquinone) and HMF were obtained from Wako Pure Chemical Industries (Osaka, Japan). XTT was purchased from Polyscience (Warrington, PA). All other reagents were of the highest grade commercially available. Milli-Q water was used in all procedures.

Milk samples Two kinds of UHT-treated milks with a fat content of $3.5 \%$ and 3.6\%, supplied by Meiji Milk Products Co., were selected as the test products for all investigations. The UHT treatment of the samples was carried out at $130^{\circ} \mathrm{C}$ for $2 \mathrm{~s}$ (Milk A) and at $140^{\circ} \mathrm{C}$ for $3 \mathrm{~s}$ (Milk B) by an indirect heat exchange apparatus containing steam-heated and watercooled capillary tubes. Milks A and B were produced from identical milk sources. Milk B was manufactured as LL milk. The package size of the milk samples was $200 \mathrm{ml}$ (Milk A) and $250 \mathrm{ml}$ (Milk B), and each sample was drawn just after opening the package. The packaged milk was stored in the dark at a given temperature. In one experiment, a commercially available pasteurized milk was used. The heat treatment of the product was carried out at $65^{\circ} \mathrm{C}$ for $30 \mathrm{~min}$ (Milk C).

Assay procedures The assay was performed in a 96-well microtiter plate using an 8-channel adjustable volume pipettor. Each well contained a given volume of $0.5 \mathrm{mM}$ XTT solution prepared with $0.2 \mathrm{M}$ potassium phosphate buffer 
containing menadione at the saturation level (ca. $0.55 \mathrm{~mm}$ ). A given amount of a milk sample was added into the well. After it was mixed in a microplate shaker for $10 \mathrm{~s}$ at a speed of 500 $\mathrm{rpm}$, the difference in the absorbance between $492 \mathrm{~nm}$ and $600 \mathrm{~nm}$ (as the reference) was read on a microplate reader MPR A4i (Tosoh, Tokyo, Japan) as the absorbance at $0 \mathrm{~min}$. Again, after $20 \mathrm{~min}$ at room temperature, the absorbance difference was read, and the increase in the absorbance difference $(\Delta A)$ was recorded as the ability of the sample to reduce $\mathrm{XTT}$.

Determination of HMF HMF was colorimetrically determined according to the method described by Keeney and Bassette (1959). In this method, bound HMF was released by heating with dilute oxalic acid. After the precipitation of the protein with trichloroacetic acid and filtering, the yellow coloring formed after the addition of thiobarbituric acid was spectrophotometrically measured at $443 \mathrm{~nm}$. Determinations were carried out in duplicate.

\section{Results and Discussion}

A turbidity appears in the reaction solution on the addition of a milk sample. The turbidity results in an apparent increase in the absorbance by lowering the transmittance. However, there was no significant difference in the apparent absorbance of the milk diluted with the buffer not containing XTT between that at $492 \mathrm{~nm}$ and at $600 \mathrm{~nm}$. The XTT formazan, which is the reduced form of XTT, had an absorption maximum in the range of $460-480 \mathrm{~nm}$, in agreement with the previous report (Roslev \& King, 1993) and showed only negligible absorption at $600 \mathrm{~nm}$. Therefore, the difference in the absorbance between $492 \mathrm{~nm}$ and $600 \mathrm{~nm}$ of a reaction mixture containing the milk sample and the XTT solution permits estimation of the formed formazan without interference from the turbidity. As was expected, no appreciable increase in the apparent absorbance by the turbidity was observed when a different volume of milk sample was added into the buffer solution (Fig. 1). The reaction mixture in which a milk sample was added into XTT solution showed a higher absorbance even at 0 min after mixing than that of the mixture containing the milk sample and the buffer

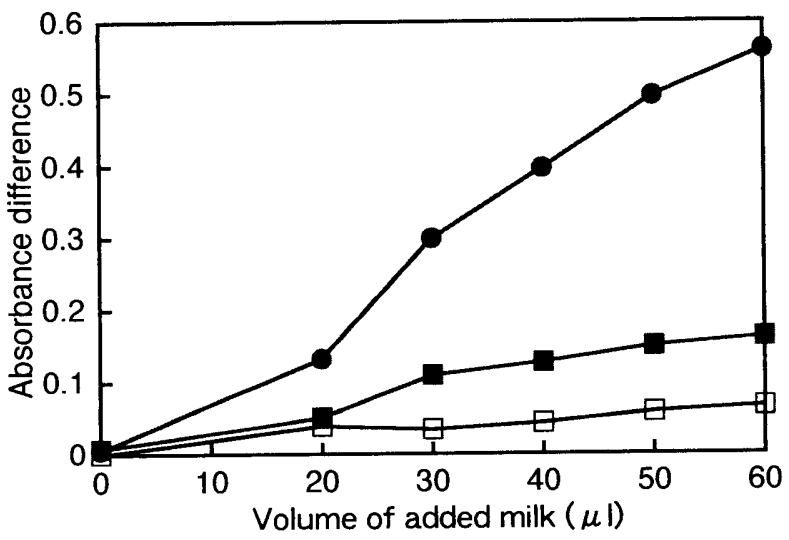

Fig. 1. Effect of the addition volume of the UHT-treated milk on the absorbance difference. The indicated volume of UHT-treated milk (Milk B) was added to $60 \mu 1$ of $0.2 \mathrm{M}$ potassium phosphate buffer $(\mathrm{pH} \mathrm{7.0)}(\mathrm{\imath}, 0 \mathrm{~min}$ after the addition) or the buffer containing $0.5 \mathrm{mM} \mathrm{XTT} \mathrm{( \square ,0} \mathrm{min;} \bullet, 20 \mathrm{~min}$ after the addition).

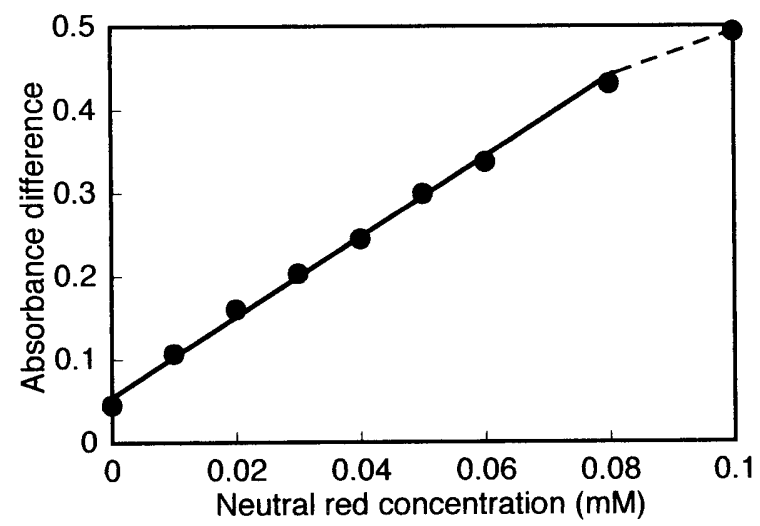

Fig. 2. Relationship between the concentration of neutral red dissolved in the mixture containing $60 \mu \mathrm{l}$ of buffer and $40 \mu \mathrm{l}$ of milk and the absorbance difference.

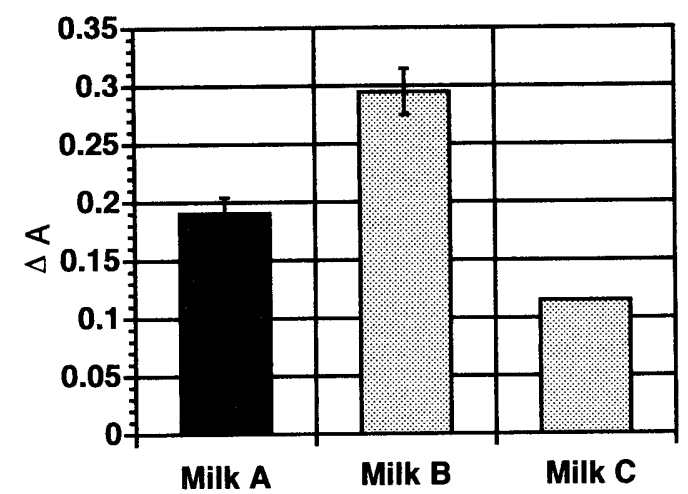

Fig. 3. Comparison of the ability of the UHT-treated milks (Milks A and B) and pasteurized milk (Milk C) to reduce XTT. Each bar for the UHT-treated milks represents the mean $\pm \mathrm{SD}$ of four different lots.

without XTT (Fig. 1). This is due to the absorption of XTT (in the oxidized form) at $492 \mathrm{~nm}$. After $20 \mathrm{~min}$, an increase in the absorbance difference of the reaction mixture was recognized dependent on the volume of the milk sample added (Fig. 1). This increase was considered to reflect the ability of the UHT-treated milk to reduce XTT.

As shown in Fig. 1, the increase in the volume of the added milk sample was expected to improve the sensitivity for detection of the reduction ability. However, the decrease in transmittance caused by the addition of milk might lead to impairment of the linear relationship between the absorbance difference and the concentration of the formazan formed. Thus, the linear range between them was examined using a solution containing $40 \mu \mathrm{l}$ of milk sample and $60 \mu \mathrm{l}$ of the buffer solution. Because the XTT formazan was not commercially available, neutral red having absorption at $492 \mathrm{~nm}$ instead of the XTT formazan was dissolved in the buffer at a given concentration. As indicated in Fig. 2, the absorbance difference of the mixture was proportional to the concentration of neutral red in the range of $0-0.08 \mathrm{mM}$ with a correlation coefficient of 0.998 , up to 0.45 as the absorbance difference. This result suggests that the linear relationship between them was retained in spite of the high turbidity of the 


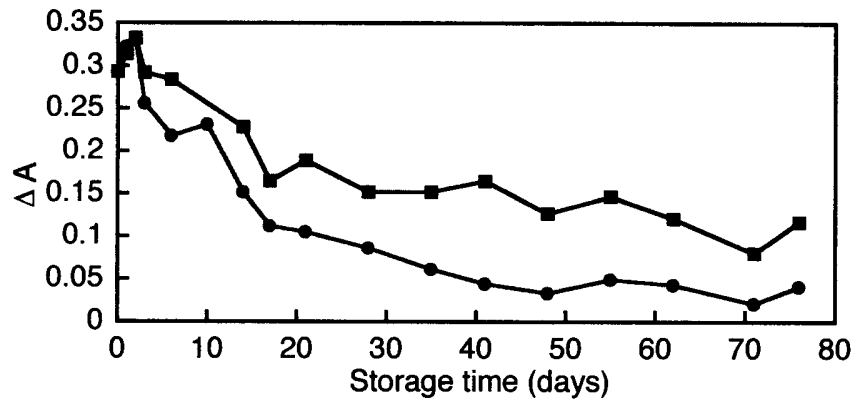

Fig. 4. Relationship between the ability of UHT-treated milk to reduce XTT $(\triangle A)$ and the storage time and temperature. UHT-treated milk (Milk B) was stored at $5^{\circ} \mathrm{C}(\boldsymbol{\square})$ or $20^{\circ} \mathrm{C}(\boldsymbol{\theta})$

reaction mixture, and the absorbance difference (0.45) was regarded as the upper limit of the linear range in the assay solution. The absorbance which the reaction mixture containing $40 \mu \mathrm{l}$ of milk sample and $60 \mu \mathrm{l}$ of XTT solution showed after a reaction time of $20 \mathrm{~min}$ was approximately 0.4 , while the reaction mixture containing a greater volume of milk showed more than 0.45 (Fig. 1). In addition, the milk sample used here (Milk B) had a relatively higher extent of UHT treatment and thus the absorbance difference which the sample showed could be regarded as the maximum value. From these results, we selected the combination of $40 \mu \mathrm{l}$ of milk sample and $60 \mu \mathrm{l}$ of XTT solution as the optimum conditions.

Using these conditions, the effects of the concentration of XTT and menadione and the reaction $\mathrm{pH}$ on the absorbance difference were examined. The results were almost the same as those in our previous paper (Ukeda et al., 1995) in spite of using a different ratio of XTT and sample solution and a different detection system. Hence the identical conditions were selected as the XTT $(0.5 \mathrm{mM})$ and menadione (the saturation level, about $0.55 \mathrm{~mm}$ ) concentration and the $\mathrm{pH}$ ( $\mathrm{pH} 7$ ).

Next the optimized microtiter plate assay was applied to two kinds of UHT-treated milk (Fig. 3). The extent of the UHT treatment is more severe in Milk B than in Milk A. Support for this was furnished by the HMF value of the milks (Milk A, $8.95 \mu \mathrm{M}$; Milk B, $12.6 \mu \mathrm{M}$ ) (Kessler \& Fink, 1986). Each $\Delta A$ obtained as the average of four lots showed a statistically significant difference between them at the $0.5 \%$ level. Similarly, the XTT assay was applied to a pasteurized milk (Milk C) (Fig. 3). The pasteurized milk sample also showed reducibility, although the value was smaller than that of UHT-treated milk. These results are in accord with our previous results obtained by the batch method (Ukeda et al, 1995). The relative standard deviation (rsd) of the present microtiter plate assay was better than $6 \%(n=10)$ with satisfactory reproducibility. However, the reproducibility was slightly inferior to that of our previous batch method ( $\mathrm{rsd}=$ 3.9\%). This may be due to imperfect mixing of milk with XTT solution in a well with such a small volume.

Figure 4 shows the change in the XTT reducibility of Milk $\mathrm{B}$ during storage. The ability decreased with storage time and temperature, consistent with the results obtained by our previous batch method (Ukeda et al., 1995). Hence, the present microtiter plate assay is also applicable to estimation of the storage period after heat treatment if the storage temperature is known or vice versa.

\section{Conclusions}

The present XTT assay is much easier and faster than the conventional methods of estimating the extent of the Maillard reaction such as the HMF and lactulose determinations. The use of a microtiter plate in the XTT reduction assay is especially advantageous because the consumption of the expensive reagent XTT is much less than that in the previously reported batch method (Ukeda et al, 1995). In addition, a higher sampling frequency is achieved. Therefore, the present method is more practical for quality control in the dairy industry.

\section{References}

Fink, R. and Kessler, H.-G. (1988). Comparison of methods for distinguishing UHT treatment and sterilization of milk. Milchwissenschaft, 43, 275-280.

Finot, P.A., Deutsch, R. and Bujard, E. (1981). The extent of the Maillard reaction during the processing of milk. Prog. Food Nutr. Sci. 5, 345-355.

Geier, H. and Klostermeyer, H. (1980). Enzymatische Bestimmung von Lactulose. Z. Lebensm. Unters. Forsch., 171, 443-445.

Keeney, M. and Bassette, R. (1959). Detection of intermediate compounds in the early stages of browning reaction in milk products. $J$. Dairy Sci., 42, 945-960.

Kessler, H.-G. and Fink, R. (1986). Changes in heated and stored milk with an interpretation by reaction kinetics. J. Food Sci, 51, 11051111

Roslev, P. and King, G.M. (1993). Application of a tetrazolium salt with a water-soluble formazan as an indicator of viability in respiring bacteria. Appl. Environ. Microbiol., 59, 2891-2896.

Ukeda, H., Goto, Y., Sawamura, M., Kusunose, H., Kamikado, H. and Kamei, T. (1995). Reduction of tetrazolium XTT with UHT-treated milk: Its relationship with the extent of heat-treatment and storage conditions. Food Sci. Technol. Int. 1, 52-57.

Walstra, P. and Jenness, R. (1984). Heating. In "Dairy Chemistry and Physics." John Wiley \& Sons, New York, pp. 162-185. 